Journal for ImmunoTherapy of Cancer

\section{PET/CT scan and biopsy-driven approach for safe anti-PD-1 therapy discontinuation in patients with advanced melanoma}

To cite: Gibney GT, Zaemes J, Shand S, et al. PET/CT scan and biopsy-driven approach for safe anti-PD-1 therapy discontinuation in patients with advanced melanoma. Journal for ImmunoTherapy of Cancer 2021;9:e002955. doi:10.1136/ jitc-2021-002955

- Additional supplemental material is published online only. To view, please visit the journal online (http://dx.doi.org/10. 1136/jitc-2021-002955).

Accepted 13 July 2021

\section{Check for updates}

(c) Author(s) (or their employer(s)) 2021. Re-use permitted under CC BY-NC. No commercial re-use. See rights and permissions. Published by BMJ.

For numbered affiliations see end of article.

\section{Correspondence to} Dr Geoffrey T. Gibney; geoffrey.t.gibney@gunet. georgetown.edu

\section{ABSTRACT}

Background Limited data exist on safe discontinuation of antiprogrammed cell death protein 1 (PD-1) therapy in responding patients with advanced melanoma. The use of 18fluorodeoxyglucose $\left({ }^{18} \mathrm{FDG}\right)$-PET/CT scan and tumor biopsy for assessment of active disease may be an effective predictive biomarker to guide such treatment decisions.

Methods A retrospective study of 122 patients with advanced melanoma treated with anti-PD-1 monotherapy or anti-PD-1/anticytotoxic T-lymphocyte-associated protein 4 combination therapy at Georgetown Lombardi Comprehensive Cancer Center was conducted. Uveal melanoma patients and those receiving concurrent experimental therapy were excluded. Baseline characteristics, treatment outcomes, and survival were analyzed. Patients who decided to come off treatment typically after 12 months using CT scan radiographic complete response (CR), ${ }^{18} \mathrm{FDG}$-PET/CT scan complete metabolic response (CMR) or tumor biopsy of a non-CR/ CMR tumor site negative for active disease (possible pathological $\mathrm{CR}$ ) were identified and compared with patients who discontinued treatment due to toxicity while their disease was in control. Event-free survival (EFS) was assessed from the last dose of anti-PD-1 therapy to progression requiring subsequent treatment (surgery, radiation, and/or systemic therapy) or referral to hospice/ death due to melanoma.

Results 24 (20\%) patients discontinued treatment by choice with no active disease and $28(23 \%)$ patients discontinued treatment due to toxicity with disease control after 12-month and 4-month median treatment durations, respectively. Similar baseline characteristics were observed between cohorts except higher prior receipt of ipilimumab (29\% vs $7 \% ; p=0.036)$ and fewer BRAF mutant positive disease ( $17 \%$ vs $41 \% ; p=0.064)$ in patients off treatment by choice. Three-year EFS rates were $95 \%$ and $71 \%$, respectively. No significant associations between EFS and sex, disease stage, lactate dehydrogenase elevation, BRAF status, prior systemic therapy, ECOG performance status, presence of brain metastases, or combination versus monotherapy were observed. Tumor biopsies led to alternative management in $3 / 10$ patients due to active metastatic melanoma or second malignancy.
Conclusions Anti-PD-1 therapy discontinuation after 12 months when no active disease is observed on CT scan, PET/CT scan or tumor biopsy may have low rates of disease relapse in patients with advanced melanoma. Biopsy of residual disease may frequently lead to a change in management. These findings are undergoing validation in the EA6192 trial.

\section{BACKGROUND}

Immune checkpoint inhibitor therapies targeting programmed cell death protein 1 (PD-1) and cytotoxic T-lymphocyte-associated protein 4 (CTLA-4) have become standard therapy options for patients with advanced unresectable stage III and IV melanoma. AntiPD-1 monotherapy (nivolumab, pembrolizumab) and combination anti-PD-1/ anti-CTLA-4 therapy (nivolumab plus ipilimumab, pembrolizumab plus ipilimumab) have demonstrated objective response rates of $45 \%-61 \%$ in the front-line setting, along with improved progression-free survival (PFS) and overall survival (OS) compared with ipilimumab monotherapy and historical data. ${ }^{1-4}$ Long-term follow-up has demonstrated 5-year OS rates of $41 \%-52 \%$. However, the optimal duration of therapy in responding patients without toxicity issues is unknown.

On the Keynote 001 study, where patients with complete response (CR) on two serial scans with pembrolizumab were permitted to discontinue treatment and follow by observation alone, the estimated 24-month diseasefree survival rate was $90 \%$ (median duration of treatment was 23 months).$^{3}$ On the Keynote 006 study, there was a preplanned discontinuation of therapy after 2 years of pembrolizumab in patients with maintained disease control (CR, partial response (PR), or stable disease $(\mathrm{SD}))$ and no dose limiting toxicities. ${ }^{4}$ 
A total of 103 patients met these criteria and the estimated 24-month PFS rate after pembrolizumab discontinuation was $78 \%$. The estimated PFS rates off treatment for patients with CR or PR were $85 \%$ and $82 \%$, respectively, and $40 \%$ in those with SD suggesting that many patients with radiographic PR and SD had no residual active tumor. It remains unclear if shorter durations of anti-PD-1 therapy in selected patients with metastatic melanoma can be equally effective and which patients with prolonged PR or SD can safely discontinue therapy. Of note, in a second-line nivolumab study in patients with advanced non-small-cell lung cancer (NSCLC) (Checkmate 153), patients with disease control randomized to continue treatment beyond 1 year had superior PFS compared with those who were assigned to discontinue therapy at the one-year time point $(\mathrm{HR}=0.42),{ }^{5}$ suggesting that at least in patients with NSCLC discontinuation of treatment without further justification may be detrimental.

Durable responses with short durations of anti-PD-1 therapy have been seen in patients with advanced melanoma who stop immunotherapy early due to toxicity. On the Checkmate 067 and 069 studies, 56/96 (58\%) of patients who discontinued nivolumab plus ipilimumab in the first 12 weeks (median treatment duration 1.4 months) due to toxicity achieved an objective response and ongoing responses were observed in $64 \%$ of these responding patients. ${ }^{6}$ Of note, there is a known association of immune toxicities and clinical benefit with antiPD-1 therapy, ${ }^{7}$ suggesting that patients with melanoma and a strong antitumor immune response with treatment may only need a brief course of therapy in order to eliminate their viable tumor cells. This is supported by neoadjuvant studies with pembrolizumab, nivolumab and nivolumab plus ipilimumab, which have demonstrated complete pathological responses in $19 \%-57 \%$ of patients with advanced locoregional melanoma after 1-3 cycles of treatment even though the actual radiologic response rate was considerably less. ${ }^{8}$

The current standard, conventional computed tomography (CT) scan, for assessing benefit likely underrepresents the number of patients with complete therapeutic response with anti-PD-1 therapies in advanced melanoma given that pathological responses occur before radiographic responses and many patients with radiographic PR and SD have durable disease control after treatment is discontinued. The use of ${ }^{18}$ fluorodeoxyglucose $\left({ }^{18} \mathrm{FDG}\right)$-positron emission tomography/CT (PET/ CT) scan and/or biopsy of residual tumors may serve as a better biomarker for assessing residual active disease and thus need for further therapy. In a study conducted by the Melanoma Institute of Australia, complete metabolic response (CMR) in tumor sites by ${ }^{18}$ FDG-PET/CT scan after 1 year of anti-PD-1/programmed cell death-ligand 1 (PD-L1) therapy was associated with superior survival compared with patients with a non-CMR. ${ }^{9}$ The subgroup of patients with a radiographic PR but with a CMR, the PFS rate was $100 \%$ at 12 months and $93 \%$ at 24 months post- ${ }^{18}$ FDG-PET/CT. Further, of those patients with PR or SD but non-CMR, the PFS rate was $49 \%$ after 24 months. This implies complete pathological response may have been achieved despite the residual radiographic and/ or metabolic findings. Data from neoadjuvant antiPD-1 studies reinforce the long-term clinical impact of complete pathological response to immunotherapy, where recurrence-free survival has been reported to be $100 \%$ at 24 months in high-risk patients. ${ }^{8}$

Given the lack of a standard of care for anti-PD-1 treatment duration and the frequency of pathological CR in patients without CR on CT scans, patients at Georgetown Lombardi Comprehensive Cancer Center have been offered a PET/CT scan after approximately 12 months of therapy to determine if tumor sites are metabolically active and if so, they are offered to undergo a biopsy of a representative site for evaluation of active residual disease. If patients are negative for active disease, they have been offered the option of being observed off active anti-PD-1 therapy. Here, we present our experience in patients with advanced melanoma treated with anti-PD-1 monotherapy or anti-PD-1/anti-CTLA-4 combination therapy to determine the frequency of residual active disease by ${ }^{18} \mathrm{FDG}$ $\mathrm{PET} / \mathrm{CT}$ scan metabolic response and/or tumor biopsy and the durability of response in patients who come off treatment by choice without evidence of active disease in comparison to patients with disease control who needed to come off treatment due to toxicity.

\section{METHODS \\ Patients and data collection}

Under an Institutional Review Board (IRB)-approved protocol at Georgetown University, clinical outcome data were collected retrospectively on patients with advanced, unresectable stage III and IV melanoma treated with antiPD-1 monotherapy (pembrolizumab or nivolumab) and combination anti-PD-1/anti-CTLA-4 therapy (nivolumab plus ipilimumab or pembrolizumab plus ipilimumab) at the MedStar Georgetown University Hospital Lombardi Comprehensive Cancer Center from 2013 to 2019. Patients with uveal melanoma were excluded as well as those with prior anti-PD-1 treatment in the adjuvant setting or receipt of concurrent experimental therapy. If patients received more than one line of anti-PD-1-based therapy, only the first line of therapy was included in the analyses. Baseline characteristics, therapeutic regimens, best overall radiographic response (provider assessed), treatment duration, the reason for treatment discontinuation, PFS, and OS were captured. Patients with disease control (CR, PR, or SD) who discontinued therapy due toxicity and by choice after a CR by CT scan or no active disease by ${ }^{18}$ FDG-PET/CT scan and/or tumor biopsy were analyzed. The elective treatment discontinuation was typically after 12 months of active therapy based on practice patterns at our cancer center, but patients receiving shorter and longer durations of treatment were included, such as where a CR or a negative tumor biopsy was 
observed prior to 12 months or patients' preference to remain on treatment longer than 12 months. Criteria for no active disease by ${ }^{18}$ FDG-PET/CT scan were concluded when no suspicious hypermetabolic lesions were identified, that is, CMR as determined by ${ }^{18}$ FDG-uptake by standard uptake value (SUV) in responding tumor lesions was $\leq \mathrm{SUV}$ of the liver. Tumor biopsy was performed in patients not achieving CMR and considered negative when no viable tumor cells were identified by H\&E or melanoma immunohistochemistry (IHC) markers (eg, S100, melan-A, or HMB-45). The finding of melanin laden or IHC positive macrophages (melanophages) in the absence of viable tumor cells was considered to be negative for residual tumor.

\section{Statistical methods}

Descriptive statistics were summarized for age, gender, primary melanoma type, BRAF status, Eastern Cooperative Oncology Group performance status (ECOG PS), American Joint Committee on Cancer (AJCC) eighth edition stage at the time of anti-PD-1 therapy, lactate dehydrogenase $(\mathrm{LDH})$, presence of brain metastases, receipt of prior therapies, and type of anti-PD-1 therapy received. For continuous variables, a Student's t-test was used for determination of statistical differences. For proportion, differences between the populations, $\chi^{2}$ tests (Pearson correlation) were used for categorical variables. Best overall response was based on provider assessment following Response Evaluation Criteria in Solid Tumours (RECIST) guidelines. ${ }^{10}$ Patients with SD on the first restaging scan but either clinical or radiographic disease progression by the next scan time point were considered progressive disease (PD) overall. Patients with unconfirmed PR were considered SD. Patients assessed to have clinical deterioration/progression without restaging scans were assigned PD. OS was defined as the duration from the date of first anti-PD-1 dose to the date of death/ hospice referral related to melanoma. Event-free survival (EFS) was defined as the duration from the date of the last anti-PD-1 dose to date of progression requiring treatment (systemic therapy, surgery, or radiation) or death/ hospice referral related to melanoma. Survival was evaluated using the Kaplan-Meier (KM) method and Cox regression. Survival differences between populations were determined using a log-rank test for KM. All statistical analyses were performed using IBM SPSS (V.24) and GraphPad Prism (V.6.0). A two-sided $\mathrm{p} \leq 0.05$ was considered statistically significant.

\section{RESULTS}

\section{Patient characteristics}

A total of 122 patients with advanced melanoma receiving standard anti-PD-1 monotherapy or anti-PD-1/antiCTLA-4 combination therapy were included in the study (online supplemental table 1). There were 46 subjects who received anti-PD-1 monotherapy and 76 who received an anti-PD-1/anti-CTLA-4 combination therapy. Most subjects had cutaneous or unknown primary $(84 \%)$, stage IV M1c/M1d disease (61\%), and ECOG PS 0-1 (94\%). Prior systemic therapy had been administered in $36 \%$ of patients. Provider assessed best overall response was $52 \%$ (CR 12\%; PR 40\%; SD 11\%; PD 38\%). Five-year landmark OS was estimated to be $58 \%$ ( $53 \%$ for the monotherapy cohort and $62 \%$ for the combination therapy cohort, $\mathrm{p}=0.052$; online supplemental figure 1 ).

Of the 28 patients considered for monitoring off treatment by choice, 22 subjects underwent PET/CT scan to evaluate for metabolic response (figure 1). Fourteen (64\%) patients had CMR. All eight non-CMR patients underwent tumor biopsy: six patients demonstrated no viable tumor cells, one patient demonstrated a new primary NSCLC (residual hypermetabolic lung mass on PET/CT scan), and one patient demonstrated a new primary colorectal cancer (residual hypermetabolic bowel lesion on PET/CT scan). Two patients with PR without a PET/CT scan underwent biopsy of a representative tumor site to evaluate for active disease-one patient demonstrated no viable tumor cells while the second patient demonstrated viable melanoma tumor cells and subsequently had treatment changed to talimogene laherparepvec (TVEC) plus anti-PD-1 therapy. In total, 3 out of 10 patients who underwent a biopsy to evaluate for active residual disease demonstrated active melanoma or another malignancy leading to a change in management.

Twenty-four patients (20\%) came off active treatment by choice after no evidence of active disease by CR on CT scan, CMR on PET/CT scan or negative tumor biopsy (figure 1). Twenty-eight patients (23\%) came off active treatment due to toxicity with disease control. These two groups were further analyzed for EFS as individual cohorts and then combined to examine EFS by baseline patient and disease characteristics. Table 1 shows the baseline characteristics for these two patient cohorts included in the EFS analyses. Overall, similar characteristics were observed between patients who came off treatment by choice with no active disease and those who came off treatment for toxicity. This included age (median 67 and 63 years, respectively), elevated LDH (29\% in each), M1c/ M1d disease ( $54 \%$ and $75 \%$, respectively), brain metastases ( $42 \%$ and $25 \%$, respectively), prior systemic therapy (33\% and 25\%) and receipt of anti-PD-1/anti-CTLA-4 combination therapy ( $58 \%$ and $75 \%$, respectively). Exceptions include fewer patients with $B R A F$ mutant melanoma ( $17 \%$ vs $41 \%, p=0.064)$ and more patients with prior ipilimumab (29\% vs $7 \%, \mathrm{p}=0.036)$ in patients off treatment by choice than patients off treatment for toxicity.

\section{Best overall response, treatment duration, and determination of no active disease}

In the cohort of patients off treatment by choice, the best overall response by CT scan was CR in 8/24 patients (33\%), PR in $15 / 24$ patients $(63 \%)$ and SD 1 patient (4\%). Median duration of treatment was 12.1 months (range 2.3-24.1 months). The patients came off treatment after demonstrating CR on CT scan alone $(n=3 / 24$; 


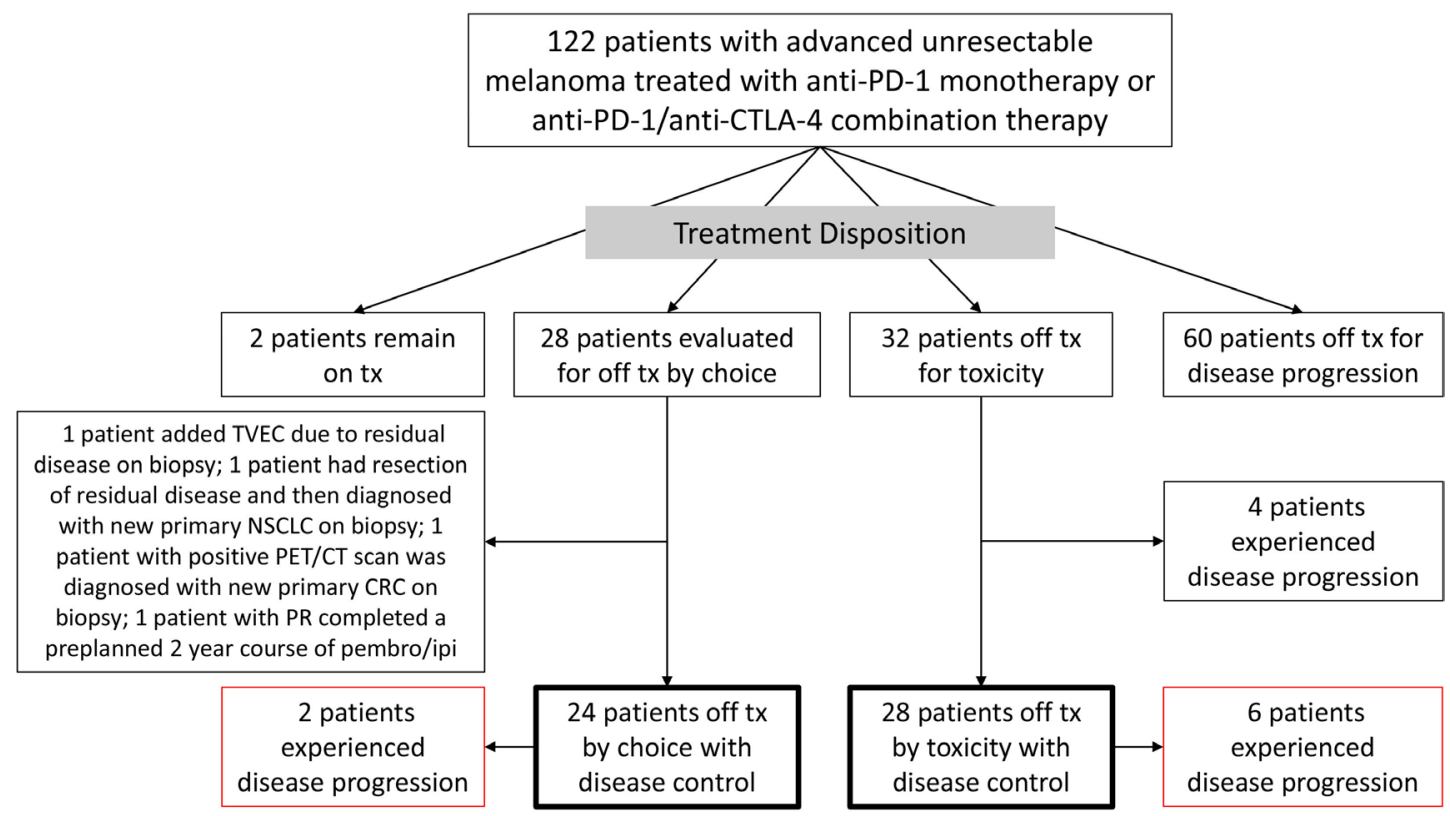

Figure 1 Outcomes for patients with advanced melanoma treated with anti-PD-1 therapy. Of 122, 24 (20\%) of patients came off treatment by choice based on no evidence of active disease by imaging and/or biopsy. Of 122, 28 (23\%) of patients came off treatment due to toxicity with disease control. CTLA-4, cytotoxic T-lymphocyte-associated protein 4; NSCLC, non-smallcell lung cancer; PD-1, programmed cell death protein 1; TVEC, talimogene laherparepvec; CRC, colorectal cancer; PR, partial response.

$13 \%)$, CMR on PET/CT scan ( $\mathrm{n}=14 / 24,58 \%)$, and nonCMR on PET /CT scan but biopsy negative $(\mathrm{n}=6 / 24 ; 25 \%)$. Note five of the patients with CMR on PET/CT scan also had a CR on CT scan. One additional patient had initial increase in nodal disease and biopsy was negative for active disease; patient elected to discontinue treatment after 2.3 months and subsequent CT scans demonstrated PR $(\mathrm{n}=1 / 24 ; 4 \%)$. Of the patients with CMR on PET/CT scan, five had CR, eight had PR, and one had SD on diagnostic CT scan. No patient with a CR on CT scan had a non-CMR PET/CT scan. For the six patients with nonCMR and negative biopsy, all demonstrated PR by diagnostic CT scan. Figure 2 illustrates a patient with a PR on CT scan and non-CMR by PET/CT scan where a biopsy was negative for viable tumor cells. Of note, the presence of melanophages was observed.

In the cohort of patients off treatment due to toxicity, the best overall response was CR in $5 / 28$ patients (18\%), PR in $22 / 28$ patients $(79 \%)$, and SD in $1 / 28$ patients $(4 \%)$. Median treatment duration in this cohort of patients was 3.7 months (range $0.7-14.0$ months).

\section{Event-free survival}

EFS was evaluated in patients who came off treatment by choice and who came off treatment due to toxicity with disease control. No significant EFS difference was observed between the two cohorts, although fewer events occurred in the cohort off treatment by choice $(p=0.160$, figure 3 ). In the patient cohort off treatment by choice, estimated 1-year, 2-year, and 3-year EFS rates were 100\%, $95 \%$, and $95 \%$, respectively (online supplemental table 2 ). Only 2 out of 24 patients had an event in this cohort. One patient had surgical resection of an isolated lymph node recurrence rendering him free of active disease and continues to be followed off treatment without recurrence for 3+ years; one patient with progression after a 3-year EFS is deceased after receiving subsequent rechallenge with nivolumab plus ipilimumab (online supplemental table 3). In the patient cohort off treatment due to toxicity, the estimated 1-year, 2-year, and 3-year EFS rates were $87 \%, 83 \%$, and $71 \%$, respectively (online supplemental table 2). There were six events in this cohort. Two patients had surgery to render them free of active disease; one is currently followed off active treatment and the second developed a progressive brain metastasis and is lost to follow-up (online supplemental table 3). Four patients received subsequent systemic therapy for melanoma. One patient was rechallenged with ipilimumab and developed recurrent immune colitis after four cycles. She demonstrated disease regression but then was started on dabrafenib/trametinib for clinical progression and had a CR. Dabrafenib/trametinib was discontinued due to toxicity, and she has been followed off active treatment for over 2.5 years. Three other patients with disease progression received pembrolizumab, none of whom responded to therapy. One patient is alive after CR to BRAF/MEK inhibitors, and two patients are deceased.

EFS associations with baseline patient characteristics and treatment were evaluated by KM and Cox regression analyses (refer to figure 4 and online supplemental table $4)$. Both cohorts of patients were combined for these analyses. A trend for association between primary tumor type and EFS was observed by KM with lower survival in patients with mucosal melanoma compared with other subgroups (figure 4A). However, the mucosal subgroup size was low 
Table 1 Baseline Patient characteristics

\begin{tabular}{|c|c|c|c|}
\hline Characteristics & Choice $(n=24)$ & Toxicity $(\mathrm{n}=28)$ & Statistics \\
\hline Median age & 67 years & 63 years & $\mathrm{P}=0.311$ (two-sided t-test) \\
\hline Male gender & $17(71 \%)$ & $17(61 \%)$ & $\mathrm{P}=0.444\left(\right.$ Pearson $\left.\chi^{2}\right)$ \\
\hline LDH elevated & $7(29 \%)$ & $8(29 \%)$ & $\mathrm{P}=0.971\left(\right.$ Pearson $\left.\chi^{2}\right)$ \\
\hline Primary tumor type & & & $\mathrm{P}=0.468\left(\right.$ Pearson $\left.\chi^{2}\right)$ \\
\hline Acral & $1(4 \%)$ & 0 & \\
\hline Mucosal & $2(8 \%)$ & $2(7 \%)$ & \\
\hline Unknown & $9(38 \%)$ & $7(25 \%)$ & \\
\hline Disease stage at tx & & & $\mathrm{P}=0.115\left(\right.$ Pearson $\left.\chi^{2}\right)$ \\
\hline Brain mets (\%) & $10(42 \%)$ & $7(25 \%)$ & $\mathrm{P}=0.202\left(\right.$ Pearson $\left.\chi^{2}\right)$ \\
\hline Prior systemic tx & $8(33 \%)$ & $7(25 \%)$ & $\mathrm{P}=0.508\left(\right.$ Pearson $\left.\chi^{2}\right)$ \\
\hline Ipilimumab & $7(29 \%)$ & $2(7 \%)$ & $\mathrm{P}=0.036\left(\right.$ Pearson $\left.\chi^{2}\right)$ \\
\hline BRAFi/MEKi & $1(4 \%)$ & $4(14 \%)$ & $\mathrm{P}=0.217\left(\right.$ Pearson $\left.\chi^{2}\right)$ \\
\hline Therapy received & & & $\mathrm{P}=0.202\left(\right.$ Pearson $\left.\chi^{2}\right)$ \\
\hline Anti-PD1/Anti-CTLA4 & $14(58 \%)$ & $21(75 \%)$ & \\
\hline Anti-PD-1 & $10(42 \%)$ & $7(25 \%)$ & \\
\hline
\end{tabular}

${ }^{*} \mathrm{~N}=3(13 \%)$ unknown BRAF status.

$\dagger \mathrm{N}=5(18 \%)$ unknown BRAF status.

CTLA4, cytotoxic T-lymphocyte-associated protein 4; LDH, lactate dehydrogenase; PD-1, programmed cell death protein 1; PS, performance status.

$(\mathrm{N}=4)$ and EFS association was only statistically significant on Cox proportion analyses when the mucosal and unknown primary subgroups were compared $(p=0.026)$. There were no significant associations between EFS and sex, stage of disease, presence of brain metastases, ECOG
PS, or prior systemic therapy (a separate EFS association analysis for the off treatment by choice cohort alone was performed and showed similar findings with the exception that the mucosal-unknown primary subgroup analysis was no longer significant as only two patients had mucosal
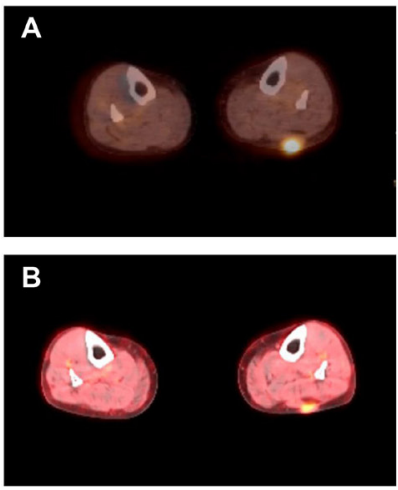
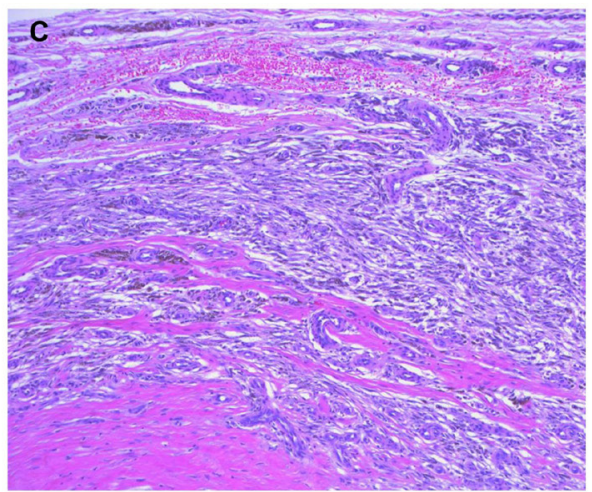

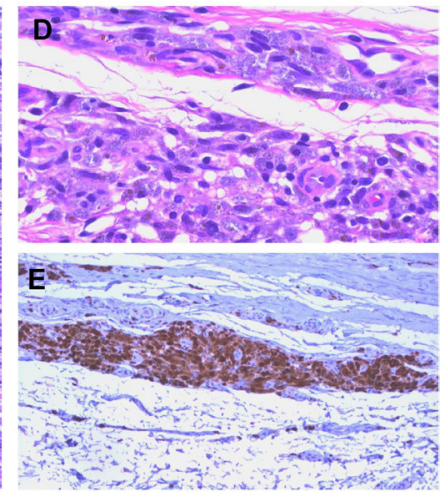

Figure 2 Example of a patient with a non-CMR on PET/CT scan but negative biopsy for viable tumor. The patient achieved a partial response to anti-PD-1 therapy. (A, B) shows a hypermetabolic subcutaneous metastasis at the left calf pretreatment and 1-year post-treatment with anti-PD-1 therapy. The patient underwent excisional biopsy of the residual left calf lesion. (C) shows pathology findings of excised lesion on H\&E stain. Fibrosis, brown pigmented cells considered melanophages and no viable tumor cells identified. (D) shows higher power view of fibrosis and melanophages. (E) shows brown stain with anti-CD68, highlighting melanophages. Additional stains showed melanophages positive for melan-A and fontana mason, but negative for iron (data not shown). CMR, complete metabolic response; PD-1, programmed cell death protein 1. 


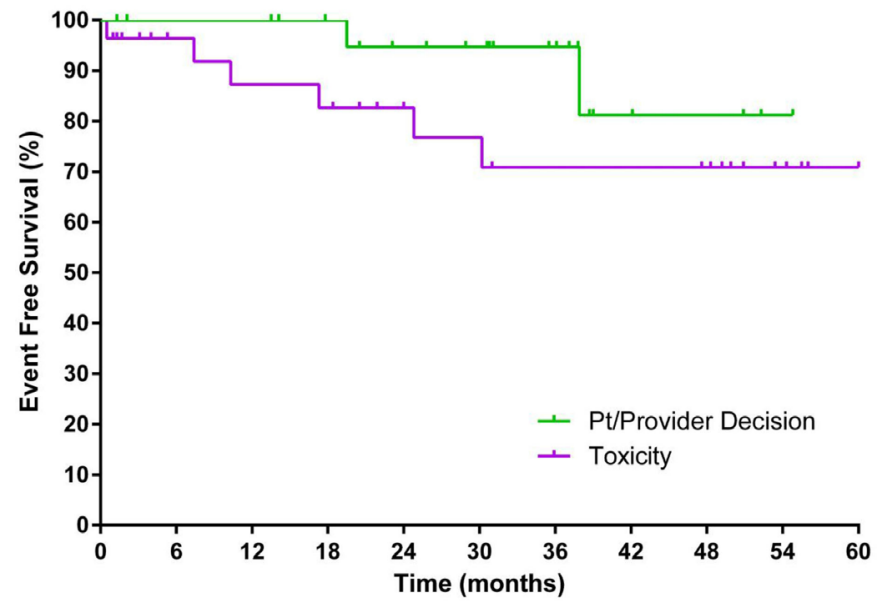

\begin{tabular}{|l|c|c|c|c|c|}
\hline & 12 mos & $\mathbf{2 4}$ mos & $\mathbf{3 6}$ mos & $\mathbf{4 8}$ mos & 60 mos \\
\hline EFS by Choice & & & & & \\
Number of events & 0 & 1 & 0 & 1 & 0 \\
Number at Risk & 24 & 22 & 16 & 10 & 3 \\
\hline EFS for Toxicity & & & & & \\
Number of events & 3 & 1 & 2 & 0 & 0 \\
Number at Risk & 28 & 19 & 15 & 11 & 10 \\
\hline
\end{tabular}

Figure 3 Event-free survival (EFS) in patients treated with anti-PD-1 therapy. Patients who discontinued treatment by choice after no evidence of active disease by CT scan, ${ }^{18}$ FDG-PET/CT scan or tumor biopsy are represented by the green survival curve $(n=24)$. Patients who discontinue treatment due to toxicity with initial disease control are represented by the purple survival curve $(n=28)$. Two events were observed in the cohort off treatment by choice and six events were observed in the cohort off treatment due to toxicity. Log-rank $p=0.160 .{ }^{18} \mathrm{FDG}$, 18fluorodeoxyglucose; PD-1, programmed cell death protein 1.

melanoma in this cohort). None of the patients with baseline ECOG PS 2-4 (6\% of all patients) achieved EFS status. EFS was numerically lower in patients treated with combination anti-PD-1/anti-CTLA-4 therapy compared with anti-PD-1 monotherapy, but this was not statistically significant (figure 4B; online online supplemental table $4)$. Of note, there was a higher representation of combination anti-PD-1/anti-CTLA-4 therapy in the total EFS data set, particularly in the toxicity cohort ( $75 \%$; table 1$)$.

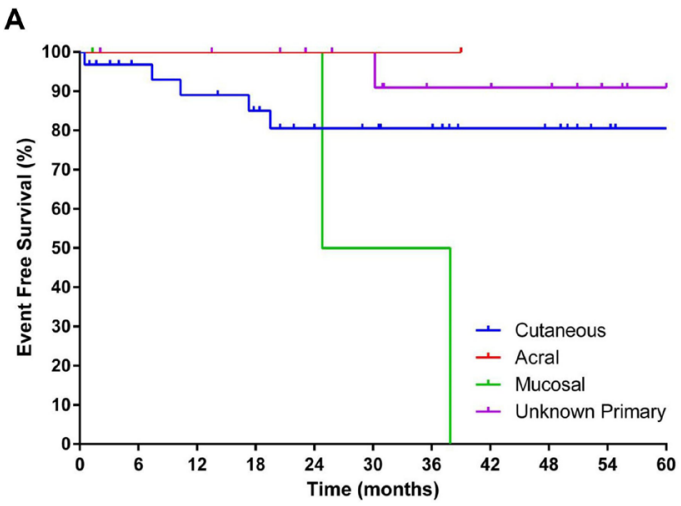

\section{DISCUSSION/CONCLUSION}

In this retrospective study, patients with advanced melanoma receiving anti-PD-1 alone and in combination with anti-CTLA-4 demonstrated efficacy similar to what has been previously reported in prospective randomized studies. ${ }^{14}$ Of note, a higher 5-year OS was observed in patients who were treated with combination anti-PD-1/ anti-CTLA-4 therapy consistent with the Checkmate 067 study. Almost half of all patients had treatment cessation in the absence of disease progression, with $23 \%$ of patients off treatment for toxicity with disease control and $20 \%$ of patients off treatment by choice without evidence of active disease. Similar high rates of EFS were seen in both cohorts with numerically greater survival rates in patients off treatment by choice. While the majority of patients received combination anti-PD-1/anti-CTLA-4 therapy in both cohorts, the radiographic CR rate was lower, and treatment duration was shorter in the patients who came off treatment due to toxicity. These factors, along with the use of immunosuppressive agents and lack of selection for absence of residual by PET/CT or biopsy, may account for the slightly lower EFS rate in the off treatment due to toxicity cohort.

Several retrospective studies of patients with advanced melanoma have also shown that elective discontinuation of anti-PD-1 therapy after treatment durations shorter than 24 months can be associated with durable off treatment survival. ${ }^{11-13}$ In one study where patients with CR, PR, or SD electively discontinued anti-PD-1 therapy after a median of 12 months, the 1-year and 2-year PFS rates after discontinuation were $90 \%$ and $71 \%$, respectively. ${ }^{12}$ Patients with CR had a significantly lower risk of disease progression compared with patients with PR or SD. Also, patients with CR who received less than 6 months of therapy had shorter PFS compared with patients with CR who received anti-PD-1 therapy for 6 months or longer. In another study of patients with CR who came off treatment after a median of 9.4 months, the 3-year PFS rate after discontinuation was $72 \% .^{11}$ The PFS rates in this second study may have been impacted by the inclusion of patients who came off treatment due to toxicity (24\%).

B

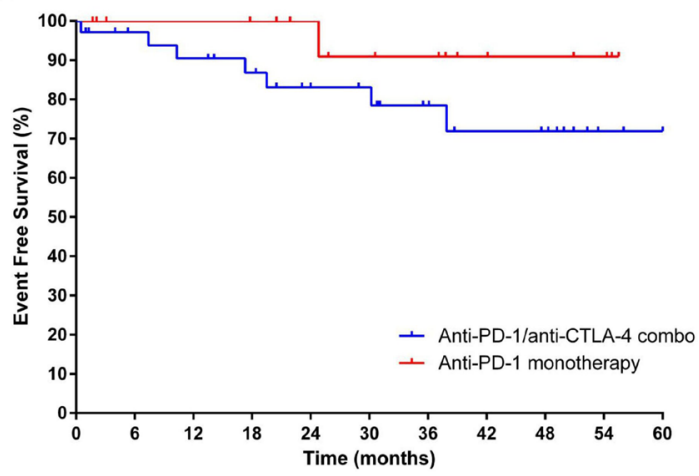

Figure 4 EFS associations with primary tumor site $(A, p=0.064)$, and receipt of anti-PD-1 monotherapy versus combination therapy $(B, p=0.195)$. EFS data for patients off treatment by choice and due to toxicity were combined for these analyses. CTLA-4, cytotoxic T-lymphocyte-associated protein 4; EFS, event-free survival; PD-1, programmed cell death protein 1. 


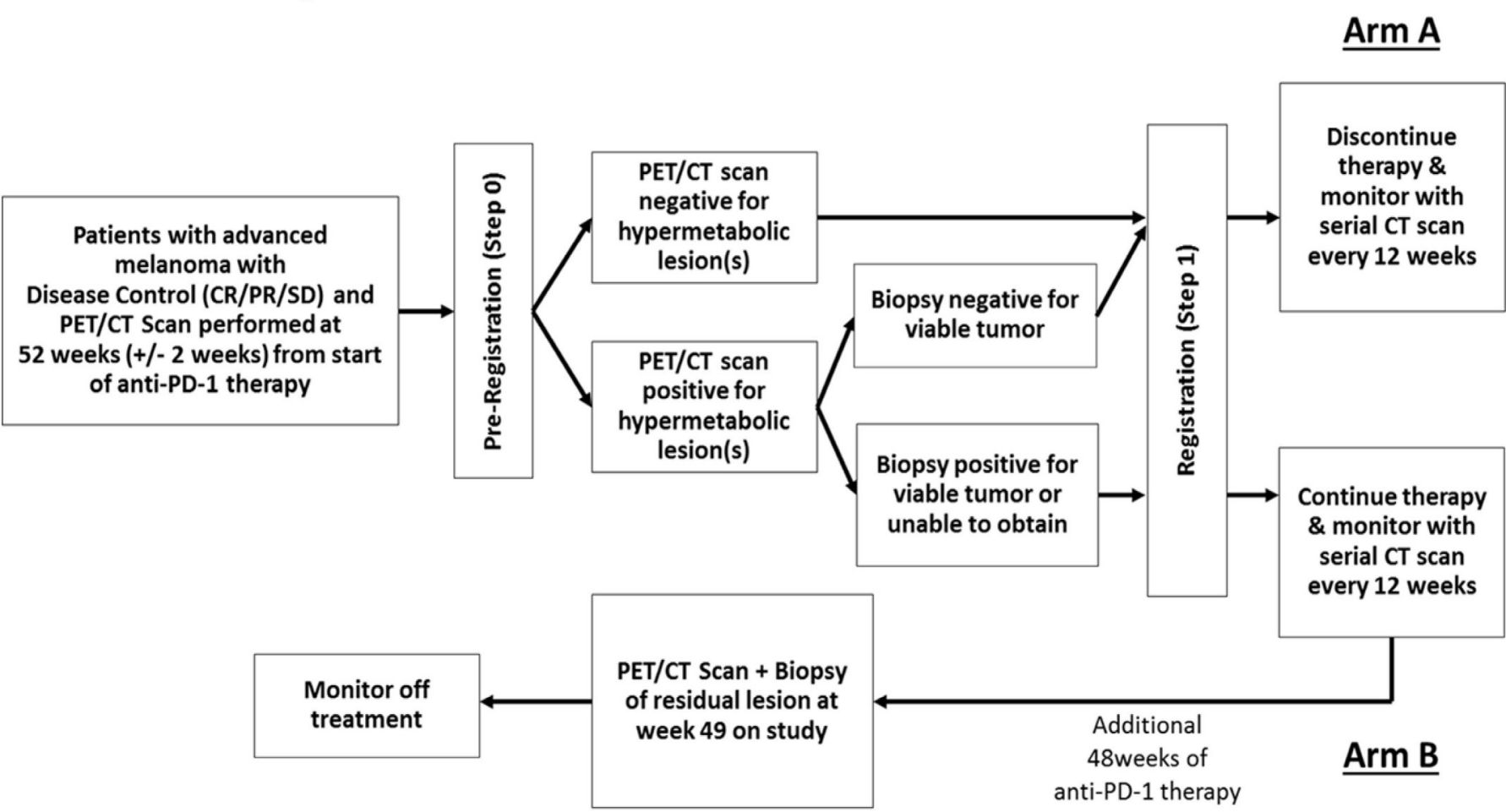

Figure 5 Study design for the ongoing EA6192 trial validating ${ }^{18} \mathrm{FDG}$-PET/CT scan and tumor biopsy as predictive biomarkers for safe anti-PD-1 therapy discontinuation in patients with advanced melanoma. ClinicalTrials.gov Identifier: NCT04462406. $\mathrm{CR}$, complete response; ${ }^{18} \mathrm{FDG}, 18$ fluorodeoxyglucose; PD-1, programmed cell death protein 1; PR, partial respons; SD, stable disease.

This assumption is supported by the higher number of progression events observed in the toxicity cohort of our study as well as in the combined Checkmate 067/069 toxicity outcome dataset. ${ }^{6}$ The more favorable PFS rate after anti-PD-1 discontinuation (EFS) in our cohort of patients off treatment by choice (2-year and 3-year EFS of 95\%) may be due to selection of patients with CMR on ${ }^{18}$ FDG-PET/CT scan and tumor biopsy showing no active disease.

The CMR rate on ${ }^{18}$ FDG-PET/CT scan in patients with $\mathrm{PR} / \mathrm{SD}$ after a median of 1 year from the start of treatment in our study $(53 \% ; n=9 / 17)$ was similar to the CMR rate reported in the Melanoma Institute of Australia study $(65 \%) .{ }^{9}$ The small difference in rates may be to the smaller sample size (random variation) and higher use of anti-PD-1/anti-CTLA-4 combination therapy with resultant increased potential for false positives due to inflammation in our study. Interestingly, 3 of 10 patients undergoing biopsy to clarify disease status had residual active melanoma or a new second malignancy. In the one patient with active residual melanoma, he was able to transition to an effective alternative immunotherapy strategy. The use of biopsy to clarify disease status in nonCMR patients appears to provide valuable management guidance. This is supported by the high rate of progression in non-CMR patients on the Melanoma Institute of Australia study despite 1 year of disease control with antiPD-1 therapy. Additional biomarkers may be important to predict survival outcomes in patients with $\mathrm{PR} / \mathrm{SD}$ and
non-CMR after 1 year of anti-PD-1 therapy, particularly when discontinuing therapy. For example, the presence of circulating tumor DNA after initiation of anti-PD-1 therapy in patients with advanced melanoma has demonstrated worse survival outcomes ${ }^{14}$ and could potentially be used as a biomarker for residual active disease.

Patient selection based on baseline characteristics may also play a role in off treatment survival. For example, patients with advanced uveal melanoma were excluded from our study based on the lower response rate and survival with anti-PD-1 therapies compared with other tumor melanoma subtypes. ${ }^{16}{ }^{17}$ In our subgroup analyses, patients with mucosal melanoma had worse EFS, although the sample size was small. This is supported by data showing lower response rates and shorter PFS with nivolumab monotherapy and in combination with ipilimumab in patients with advanced mucosal melanoma versus cutaneous melanoma. ${ }^{18}$ Other subgroups previously shown to have worse survival outcomes with antiPD-1 therapy, such as those with elevated LDH and M1c disease, ${ }^{1}$ were not statistically significant in our study for associations with EFS. This suggests that once patients show no residual disease on PET/CT or biopsy after anti-PD-1 therapy, baseline (pretreatment) poor prognostic factors may no longer be applicable. The additional comparison for receipt of anti-PD-1 monotherapy versus anti-PD-1/anti-CLTA-4 combination therapy showed no negative impact on the EFS outcomes with anti-PD-1 monotherapy. In fact, there was only one event 
after stopping therapy observed in patients treated with anti-PD-1 monotherapy compared with the seven events observed in patients treated with nivolumab plus ipilimumab. The higher rate of events in patients receiving combination anti-PD-1/anti-CTLA-4 therapy may have been due to the higher representation $(75 \%)$ in the treatment discontinuation due to toxicity cohort where treatment duration was shorter and patients may not have been free of active disease at time of discontinuation. However, this warrants further investigation.

While the probability of progression off treatment after discontinuation by choice or due to toxicity was low in our study, no patients had responses to rechallenge with antiPD-1 therapy (0/4 patients). Only one of these relapsed patients was in the treatment discontinuation by choice cohort. In other studies, response rates to rechallenge with anti-PD-1 based therapies have ranged from $15 \%$ to $54 \% .{ }^{411} 12$ It remains unclear if long-term outcomes would be different if these patients remained on continuous anti-PD-1 therapy (ie, beyond 12 or 24 months). Other prospective studies have demonstrated progression events occurring after 12 months despite continuous antiPD-1 therapy. ${ }^{14}$ This suggests that a subset of responding patients likely develop areas of subclinical resistant disease in the first 12 months of anti-PD-1 therapy and will eventually exhibit disease progression requiring alternative treatment options perhaps irrespective of whether they remain on therapy or not.

Early safe discontinuation of anti-PD-1 therapy has potential safety and cost benefits to patients. Late onset adverse events (AEs) are observed in patients, particularly when remaining on long-term anti-PD-1 therapy. In a retrospective study of 110 patients with advanced melanoma treated with at least 2 years of nivolumab or pembrolizumab, $43 \%$ of patients experienced late onset AEs, including $4 \%$ of all patients requiring hospitalization. ${ }^{19}$ Increased number of doses and duration of treatment were associated with occurrence of late-onset AEs, most frequently cutaneous, gastrointestinal and neuromuscular AEs. In addition, the financial burden on patients and the healthcare system increases with prolonged courses of anti-PD-1 therapy. This includes the cost of the anti-PD-1 agent and its administration, along with $\mathrm{AE}$ management. For example, the average monthly cost of each nivolumab and pembrolizumab treatment (drug plus administration) has been previously estimated to be US $\$ 13,736$ and US $\$ 13,387$, respectively. ${ }^{20}$ The cost for an additional year of nivolumab would be US $\$ 164,832$ and US $\$ 160,644$, respectively. Similar costs would be incurred with the larger doses with more extended dosing intervals for these two agents currently being used (current wholesale acquisition list price for nivolumab $480 \mathrm{mg}$ every 4weeks is US $\$ 13,358$ and pembrolizumab $400 \mathrm{mg}$ every 6 weeks is US\$20, 135). ${ }^{21}{ }^{22}$ Such expenses dwarf the costs of a single ${ }^{18}$ FDG-PET/CT scan at 12 months and biopsy in non-CMR patients (typically under US $\$ 10,000$ total) that would occur in $10 \%-20 \%$ of patients receiving anti-PD-1 therapies using the strategy examined here for safe discontinuation. Stopping treatment also untethers patients from frequent physician visits and often allows more freedom and confidence to return to their normal precancer lives and activities.

Based on these data and the potential benefit to patients of shorter anti-PD-1 therapy durations and longer time free from treatment, validation for the use of ${ }^{18}$ FDG-PET/ CT scan and tumor biopsy for assessing residual disease after 12 months of anti-PD-1 therapy and identifying patients at low relapse risk with treatment discontinuation is warranted. This approach for safe discontinuation of anti-PD-1 therapy is currently under investigation with the ongoing ECOG-ACRIN study, EA6192 (ClinicalTrials. gov Identifier: NCT04462406; figure 5).

\section{Author affiliations}

${ }^{1}$ Lombardi Comprehensive Cancer Center, MedStar Georgetown University Hospital, Washington, District of Columbia, USA

${ }^{2}$ Charleston Oncology, Charleston, South Carolina, USA

${ }^{3}$ Memorial Sloan Kettering Cancer Center, New York, New York, USA

${ }^{4}$ Moffitt Cancer Center, Tampa, Florida, USA

${ }^{5}$ MedStar Georgetown University Hospital, Washington, District of Columbia, USA

${ }^{6}$ Inform Diagnostics, Irving, Texas, USA

${ }^{7}$ Georgetown University Medical Center, Washington, District of Columbia, USA

${ }^{8}$ MedStar Washington Hospital Center, Washington, District of Columbia, USA

${ }^{9}$ Department of Oncology, Georgetown University, Washington, District of Columbia, USA

\section{Twitter David Swoboda @Mdswoboda}

Contributors GTG contributed in development of the research project, data collection, analyses, interpretation of the data, and manuscript writing. JZ and SS contributed in data collection, analyses, interpretation of the data, and manuscript writing. NJS and AR contributed in data collection, interpretation of the data, and manuscript writing. DS contributed in data collection interpretation of the data, and manuscript writing. KG contributed in data collection and manuscript writing. VP-R, MJR and WBA-R contributed in interpretation of the data and manuscript writing. SR contributed in development of the research project, interpretation of the data and manuscript writing. MBA contributed in development of the research project, data analyses, interpretation of the data, and manuscript writing. All authors support the submission of the manuscript for publication.

Funding Funding support for the article processing charge provided by the Sher Grant to the Georgetown-Lombardi Comprehensive Cancer Center.

Competing interests GTG has served as a consultant for Novartis, Bristol Myers Squibb, Array Biopharma, Merck, Regeneron, Exicure and Sapience Therapeutics, and has received institutional research support from Exelixis and Lucerno Dynamics. MBA has served as an advisor for Arrowhead, Aveo, Bristol Myers Squibb, Eisai, Elpis, Genetech/Roche, Leads, Merck, Novartis, Pfizer, Pneuma, Pyxis Oncology, Werewolf and as a consultant to Adagene, Agenus, Apexigen, Exelixis, Idera, ImmunoCore, lovance, and Neoleuken.

Patient consent for publication Not required.

Ethics approval This retrospective study was conducted under an Institutional Review Board (IRB) approved protocol at Georgetown University. Waiver for consent was granted by the IRB.

Provenance and peer review Not commissioned; externally peer reviewed.

Data availability statement Data are available on reasonable request. Data and materials used in this study are available by communication with the corresponding author.

Supplemental material This content has been supplied by the author(s). It has not been vetted by BMJ Publishing Group Limited (BMJ) and may not have been peer-reviewed. Any opinions or recommendations discussed are solely those of the author(s) and are not endorsed by BMJ. BMJ disclaims all liability and responsibility arising from any reliance placed on the content. Where the content includes any translated material, BMJ does not warrant the accuracy and reliability of the translations (including but not limited to local regulations, clinical guidelines, 
terminology, drug names and drug dosages), and is not responsible for any error and/or omissions arising from translation and adaptation or otherwise.

Open access This is an open access article distributed in accordance with the Creative Commons Attribution Non Commercial (CC BY-NC 4.0) license, which permits others to distribute, remix, adapt, build upon this work non-commercially, and license their derivative works on different terms, provided the original work is properly cited, appropriate credit is given, any changes made indicated, and the use is non-commercial. See http://creativecommons.org/licenses/by-nc/4.0/.

\section{ORCID iDs}

Geoffrey T. Gibney http://orcid.org/0000-0002-5401-8498

Suthee Rapisuwon http://orcid.org/0000-0002-1389-925X

Michael B. Atkins http://orcid.org/0000-0003-3901-9924

\section{REFERENCES}

1 Larkin J, Chiarion-Sileni V, Gonzalez R, et al. Five-Year survival with combined nivolumab and ipilimumab in advanced melanoma. $N$ Engl J Med 2019;381:1535-46.

2 Long GV, Atkinson V, Cebon JS. Long-term follow-up of standarddose pembrolizumab plus Reduced-Dose ipilimumab in 153 patients with advanced melanoma: KEYNOTE-029 1B. Pigment Cell \& Melanoma Res 2018.

3 Robert C, Ribas A, Hamid O, et al. Durable complete response after discontinuation of pembrolizumab in patients with metastatic melanoma. J Clin Oncol 2018;36:1668-74.

4 Robert C, Ribas A, Schachter J, et al. Pembrolizumab versus ipilimumab in advanced melanoma (KEYNOTE-006): post-hoc 5-year results from an open-label, multicentre, randomised, controlled, phase 3 study. Lancet Oncol 2019;20:1239-51.

5 Spigel DR, McCleod M, Hussein MA. CheckMate153: randomized results of continuous vs 1-year Fixed-Duration nivolumab in patients with advanced non-small cell lung cancer. Ann Oncol 2017;28:3182.

6 Schadendorf D, Wolchok JD, Hodi FS, et al. Efficacy and safety outcomes in patients with advanced melanoma who discontinued treatment with nivolumab and ipilimumab because of adverse events: a pooled analysis of randomized phase II and III trials. J Clin Oncol 2017;35:3807-14.

7 Freeman-Keller M, Kim Y, Cronin H, et al. Nivolumab in resected and unresectable metastatic melanoma: characteristics of immunerelated adverse events and association with outcomes. Clin Cancer Res 2016;22:886-94.
8 Menzies AM, Rozeman EA, Amaria RN, et al. Pathological response and survival with neoadjuvant therapy in melanoma: a pooled analysis from the International neoadjuvant melanoma Consortium (INMC). JCO 2019;37:9503

9 Tan AC, Emmett L, Lo S, et al. FDG-PET response and outcome from anti-PD-1 therapy in metastatic melanoma. Ann Oncol 2018;29:2115-20.

10 Eisenhauer EA, Therasse P, Bogaerts J, et al. New response evaluation criteria in solid tumours: revised RECIST guideline (version 1.1). Eur J Cancer 2009;45:228-47.

11 Betof Warner A, Palmer JS, Shoushtari AN, et al. Long-Term outcomes and responses to retreatment in patients with melanoma treated with PD-1 blockade. J Clin Oncol 2020;38:1655-63.

12 Jansen YJL, Rozeman EA, Mason R, et al. Discontinuation of antiPD-1 antibody therapy in the absence of disease progression or treatment limiting toxicity: clinical outcomes in advanced melanoma. Ann Oncol 2019;30:1154-61.

13 Pokomy R, McPherson JP, Grossman KF. Clinical outcomes with early-elective discontinuation of PD-1 inhibitors at one year in patients with metastatic melanoma. J Clin Oncol 2020;38:10048.

14 Lee JH, Long GV, Boyd S, et al. Circulating tumour DNA predicts response to anti-PD1 antibodies in metastatic melanoma. Ann Oncol 2017;28:1130-6.

15 Seremet T, Jansen Y, Planken S, et al. Undetectable circulating tumor DNA (ctDNA) levels correlate with favorable outcome in metastatic melanoma patients treated with anti-PD1 therapy. J Trans/ Med 2019;17:303.

16 Algazi AP, Tsai KK, Shoushtari AN, et al. Clinical outcomes in metastatic uveal melanoma treated with PD-1 and PD-L1 antibodies. Cancer 2016;122:3344-53.

17 Rossi E, Pagliara MM, Orteschi D, et al. Pembrolizumab as firstline treatment for metastatic uveal melanoma. Cancer Immunol Immunother 2019;68:1179-85.

18 D'Angelo SP, Larkin J, Sosman JA, et al. Efficacy and safety of nivolumab alone or in combination with ipilimumab in patients with mucosal melanoma: a pooled analysis. J Clin Oncol 2017;35:226-35.

19 Nardin C, Dalle S, Leccia MT, et al. Long-term immune-related adverse events under PD-1 inhibitors: a multicenter prospective cohort study (MELBASE). J Clin Oncol 2020;38:10057.

20 Tarhini A, McDermott D, Ambavane A, et al. Clinical and economic outcomes associated with treatment sequences in patients with BRAF-mutant advanced melanoma. Immunotherapy 2019;11:283-95

21 keytruda. Available: https://www.keytruda.com/financial-support/ [Accessed 7 Oct 2021].

22 Bristol-Myers Squibb pricing information. Available: https://www. bmspricinginformation.com/opdivo [Accessed 7 Oct 2021]. 